\title{
Prof. Dr. Dr. Juliane Kokott, Generalanwältin am EuGH
}

\author{
djb-Frauen machen Karriere - wir stellen sie vor
}

Das Interview führte Margarete Hofmann, Vorsitzende der RG Brüssel im djb, Referatsleiterin, EU-Kommission, Brüssel am 25. Juni 2009 in Luxemburg.



Foto: ALEXANDER Louvet, 4/6 EVEnT

Liebe Frau Professorin Kokott, ich freue mich sehr, dass wir im Anschluss an Ihren Vortrag am 4. Juni 2009 in Brüssel in der Bayerischen Landesvertretung noch ein Interview zusammen machen können. Ich habe viele Fragen:

Sie haben eine glänzende juristische Karriere gemacht. Haben Sie Ihren Berufsweg vorab genau geplant, wollten Sie beispielsweise schon zu Beginn Ihres Jurastudiums Professorin werden?

Der Wunsch, Professorin zu werden, kam im ersten Semester beim Verfassen der ersten Seminararbeit. Ich habe damals bemerkt, dass die wissenschaftliche Arbeit mir viel Spaß macht und dass ich mir sehr gut vorstellen könnte, dies langfristig zu tun. Auch Dissertation und Habilitation habe ich versucht, in vernünftigen Zeiträumen fertig zu stellen. Mein Ziel war, einen Lehrstuhl zu bekommen. Aber ich wusste auch genau, dass man sich nicht fest darauf verlassen kann, dann wirklich einen Lehrstuhl zu erhalten. Ich habe deshalb versucht, eini- germaßen jung zu habilitieren, so dass ich hilfsweise auch noch in anderen Berufen hätte gut unterkommen können.

Sie haben Ihrem beruflichen Werdegang schon frühzeitig eine internationale Ausrichtung gegeben, insbesondere durch Forschungsaufenthalte in den USA. Hat dies Ihre Karriere entscheidend beeinflusst?

Ich denke, dass die Auslandsaufenthalte durchaus meine Karriere entscheidend beeinflusst haben. Sehen Sie, jetzt bin ich Generalanwältin am Gerichtshof der Europäischen Gemeinschaften. Voraussetzung für eine sinnvolle Tätigkeit hier ist, dass man gut französisch spricht. Bei meinem ersten längeren Auslandsaufenthalt, nämlich an der Universität Genf in der französischsprachigen Schweiz, habe ich gelernt, auch auf Französisch schriftlich und juristisch zu arbeiten. Weitere Auslandsaufenthalte folgten. In Washington habe ich beim jetzigen amerikanischen Mitglied des Internationalen Gerichtshofs/Den Haag, Herrn Professor Dres. Thomas Buergenthal, studiert und später an der Harvard Universität in Cambridge/Massachusetts. Das macht sich einfach gut auf dem Lebenslauf, ein Doktortitel von Harvard und ein LL.M. So konnte ich als junge Frau meine Fähigkeiten „schwarz auf weiß“ beweisen. LL.M.-Titel werden im Übrigen auch von Großkanzleien gut gewürdigt. Des Weiteren bekommt man natürlich eine andere, weitere Perspektive und einen Hintergrund auch in anderen Rechtskulturen. Dies ist hier am Gerichtshof der Europäischen Gemeinschaften zuträglich. Aber auch bei meiner Lehrtätigkeit an der Universität, insbesondere an der Universität St. Gallen, konnte ich auf dieser Grundlage rechtsvergleichende Kurse anbieten, die guten Zulauf hatten. Auch konnte ich aufgrund meiner internationalen Ausbildung in Washington in einer großen Kanzlei und an der Universität Berkeley als „Visiting Professor“ arbeiten. Und schließlich hat es mir und unserer Familie viel Freude bereitet, diese internationalen Einblicke zu erhalten.

Hatten Sie Vorbilder, die Sie gefördert und an denen Sie sich orientiert haben?

Ich hatte einige sehr gute Lehrer, darunter natürlich mein Doktorvater, Professor Dres. Karl Doehring, der gleichzeitig einen Lehrstuhl an der Universität Heidelberg hatte und Direktor des 
Max-Planck-Instituts für ausländisches öffentliches Recht und Völkerrecht in Heidelberg war. Er hat mir von vornherein diese Leistungen, Dissertation und Habilitation, zugetraut. Das ist ganz wichtig, denn es ist ja doch immer noch etwas Besonderes zu habilitieren. Außerdem war ich die erste Frau, die in Heidelberg im Staatsrecht habilitiert hat. Zu meiner Zeit konnte man die Staatsrechtslehrerinnen, die Mitglied der Staatsrechtslehrervereinigung sind, an einer Hand abzählen. Da war es schon wichtig, jemanden zu haben, der meine angestrebte Staatsrechtslehrerlaufbahn nicht ganz sonderbar, nicht ganz außergewöhnlich und quasi unmöglich findet. Ich hatte meine Habilitationspläne ja schon gehabt, als ich nur erst Referendarin war und noch nicht mal das zweite Staatsexamen hatte. Vor Herrn Professor Dres. Doehring gab es Herrn Professor Dres. Tom Buergenthal, den ich schon erwähnt habe. Er war damals Präsident des Interamerikanischen Gerichtshofs für Menschenrechte. Tom Buergenthal hatte mich ermutigt, mich am MaxPlanck-Institut/Heidelberg zu bewerben. Das war für mich als einfache Studentin auch noch etwas Besonderes. Wenn man Menschen trifft, die Vertrauen in einen setzen und mit Überzeugung sagen: „Aus Dir wird einmal etwas“, so bildet dies ein gutes Fundament.

Welche persönlichen Eigenschaften sind aus Ihrer Sicht für eine erfolgreiche Karriere wesentlich? Sind es bei Frauen andere als bei Männern?

Wesentlich ist, dass man überhaupt Karriere machen möchte, mit allen Konsequenzen, und dass man ein klares Ziel vor Augen hat. Wenn man nicht so genau weiß, ob man z.B. Germanistik, Anglistik, Jura, Wirtschaft oder überhaupt studieren sollte, was für ein Beruf oder welche Art von Tätigkeiten und Leben einem im Übrigen erstrebenswert erscheint, dann wird man nicht bereit sein, viel Arbeit oder Energie zu investieren. Denn meistens fällt einem eine Karriere nicht einfach in den Schoß. Es braucht also Motivation, Interesse und Wissen, was man denn möchte. Dies gilt wohl für Männer und Frauen gleichermaßen. Vielleicht ist es allerdings so, dass Frauen noch immer differenzierter darüber nachdenken, was für eine Art von Leben sie eigentlich wollen, und dass für Männer das Karriereziel näher liegt und weniger hinterfragt wird.

Der prozentuale Anteil von Frauen in Führungspositionen ist in Deutschland immer noch vergleichsweise niedrig. Meinen Sie, dass eine Frauenquote hier Abhilfe schaffen kann?

Es gibt immer noch viel zu wenige Frauen in Führungspositionen. In bestimmten Bereichen könnten Quoten ein probates Mittel sein um sicherzustellen, dass qualifizierte Frauen auch tatsächlich Zugang zu Führungspositionen erhalten.

Auch am EuGH ist der Frauenanteil noch sehr gering: von den 27 Richterposten sind gerade mal drei mit Frauen besetzt, d.h. magere 11,1 Prozent! Von den acht Generalanwälten/ innen sind (immerhin) drei Frauen. Ist das ein Thema in Ihrem europäischen Umfeld, wie gehen insbesondere die weiblichen Mitglieder des EuGH damit um?
Juliane Kokott, geboren 1957 in Frankfurt am Main, studierte Rechtswissenschaften an den Universitäten in Bonn, wo sie wissenschaftliche Hilfskraft bei Prof. Dr. iur. Christian Tomuschat war, und Genf. Ihren Aufenthalt als Fulbright-Stipendiatin an der American University, Washington D.C. schloss sie mit dem LL.M. ab. Parallel dazu arbeitete sie als Assistentin am Interamerikanischen Gerichtshof. 1985 folgte ihre Promotion zum Dr. iur. utr. an der Universität Heidelberg mit der Arbeit „Das interamerikanische System zum Schutz der Menschenrechte“. Außerdem erwarb sie das Diplom der Académie Internationale de Droit Constitutionnel in Tunis.

Teil ihres Referendariats am Landgericht Heidelberg war eine Station am Bundesverfassungsgericht. Neben dem Referendariat war sie am Max-Planck-Institut für ausländisches öffentliches Recht und Völkerrecht beschäftigt. Nach dem zweiten Staatsexamen 1987 ermöglichte ihr die Otto-Hahn-Medaille der Max-Planck-Gesellschaft für den wissenschaftlichen Nachwuchs einen Forschungsaufenthalt an der Harvard Law School inklusive Promotion im Jahre 1990 zum Doctor of Juridical Sciences (S.J.D.) der Harvard Universität. 1991 schloss sie ihre Habilitation ab. Die Juristische Fakultät der Universität Heidelberg verlieh ihr die „venia legendi” für deutsches und ausländisches öffentliches Recht, Völkerrecht und Europarecht.

Ihre beruflichen Stationen sind zahlreich: Sie war Referentin u.a. für europäisches Gemeinschaftsrecht am Max-Planck-Institut für ausländisches öffentliches Recht und Völkerrecht; „Visiting Professor" für das Recht der Europäischen Gemeinschaften an der University of California - Berkeley, Boalt Hall School of Law (1991); Professorin an den Universitäten Augsburg, Heidelberg und Düsseldorf; Stellvertretende Richterin am Vergleichs- und Schiedsgerichtshof der OSZE; Mitglied/stellvertretende Vorsitzende des Wissenschaftlichen Beirats „Globale Umweltveränderungen” (WBGU) der Bundesregierung (1996 bis 2003). Von 1999 bis 2003 war sie Lehrstuhlinhaberin für Völkerrecht, Internationales Wirtschaftsrecht und Europarecht an der Juristischen Fakultät der Universität St. Gallen. Direktorin des Instituts für Europäisches und Internationales Wirtschaftsrecht der Universität St. Gallen war sie von 2000 bis 2003 , seit 2001 ist sie stellvertretende Direktorin des Master of Business Law-Programms der Universität St. Gallen.

Seit 7. Oktober 2003 ist sie als dritte Frau in der Geschichte des EuGH Generalanwältin am Gerichtshof der Europäischen Gemeinschaften, Luxemburg. Die im Rat vereinigten Vertreter der Mitgliedstaaten haben am 25. Februar 2009 beschlossen, dass sie ein neues Mandat als Generalanwältin beginnend am 7. Oktober 2009 bis zum 6. Oktober 2015 erhält.

Juliane Kokott ist verheiratet und Mutter von sechs Kindern.

Die weiblichen Mitglieder des EuGH haben sich wahrscheinlich im Laufe ihrer Karriere schon dran gewöhnt, als Frauen in der Minderheit zu sein. Ich komme, wie gesagt, aus der Universitätswelt. Da war es mindestens genauso schlimm. Hier am Gerichtshof wird die Frauenfrage eher weniger diskutiert, weil wir ja von den Mitgliedstaaten ernannt werden. Wir können da nicht sehr viel einwirken. Wir können uns jedoch darum bemühen, gute weibliche Mitarbeiter einzustellen. Unter den Mitarbeitern ist die Frauenquote besser. 
Sie werden oft als juristische Vorzeigefrau, gar als „Superfrau" bezeichnet, die eine brillante und scheinbar mühelose Karriere gemacht hat und „daneben“ noch sechs Kinder auf die Welt gebracht hat. Stören Sie solche Etikettierungen, die oft auch mit einer mehr oder minder versteckten Kritik an beruflich erfolgreichen Müttern einhergehen?

Mühelos? Grundlage von Karrieren sind nach meinem Eindruck regelmäßig viel Arbeit und Disziplin. Allerdings merkt man die Arbeit nicht so, wenn sie einem Spaß macht. Die Etikettierung als „Superfrau“ ist nach Journalistenart plakativ. Ich denke, diejenigen, die ihre Aufgabe mit Spaß gerne und gut machen, sind meistens irgendwie „super“. Für mich wäre es wahrscheinlich schwieriger, hauptberuflich Hausfrau zu sein und meine sechs Kinder mit meinem Mann ausschließlich selbst zu erziehen und zu versorgen und dazu noch den großen Haushalt vollständig alleine zu erledigen! Und an die Alternative, mich ohne Kinder vollständig auf den Beruf zu konzentrieren, mag ich schon gar nicht denken, weil sie mir nicht gefällt.

Wie verbinden Sie ganz persönlich und ganz konkret Beruf und (Groß-) Familie? Werden solche Fragen auch Ihrem Mann gestellt?

Ich verbinde Beruf und Familie so wie das andere Leute auch machen. Da bin nicht die einzige: Von den sechs weiblichen Mitgliedern des Europäischen Gerichtshof haben immerhin fünf Kinder - das ist überdurchschnittlich. Auch meine Assistentinnen haben überwiegend Kinder. Immerhin gibt es ja Wochenenden, Ferien, Abende, mitunter Mittagessen, moderne Kommunikationsmittel usw. Des Weiteren haben die Kinder auch ihre eigenen Leben: von der Krabbelgruppe, über Kindergarten bis zur Schule, Freunde und Freundinnen, befreundete Familien, Sport, Musik und so fort. Ja, ähnliche Fragen werden auch meinem Mann gestellt werden, bloß eher privat als von Journalisten. Im Übrigen wohnt die Mutter meines Mannes um die Ecke und mittlerweile unterstützen uns regelmäßig Studentinnen und eine Reinemachefrau bei der Betreuung der Kinder und im Haushalt. Mein Mann hätte einen Preis verdient, weil er diese Personen alle koordiniert. Die Studentinnen bleiben meist mehrere Jahre bei uns und können den Kindern besser Fragen bei den Hausaufgaben oder beim Musizieren beantworten als die von der Bindungsforschung wohl befürwortete einzige Beziehungsperson. Hauptbeziehungspersonen bleiben bei uns immer noch die Eltern. Im Übrigen bleibt bei einer Großfamilie das System ja stabil. Anders verhält es sich bei einer Familie mit nur einem Kleinkind und beruflich sehr stark eingespannten Eltern.

Können Sie uns kurz das der deutschen Rechtsordnung unbekannte System der Generalanwaltschaft beim Europäischen Gerichtshof erklären? Wie ist das Verhältnis der Generalanwältinnen und -anwälte und der EuGH-Richterinnen und -Richter untereinander?

Das System der Generalanwälte stammt aus dem französischen Recht. Dort heißen die Generalanwälte „rapporteurs publics“, also öffentliche Berichterstatter. Das ist eine zutreffende Bezeichnung. Wir veröffentlichen einen Entscheidungsentwurf, dem der Gerichtshof in ca. 80 Prozent der Fälle folgt. Die Generalanwälte sind unabhängige Mitglieder des Gerichts, haben denselben Status, die gleiche Mandatslänge und die gleiche Ernennungsweise wie die Richter. Richter wie Generalanwälte sind Mitglieder des Gerichtshofs. Bis zur mündlichen Verhandlung gibt es eine Kooperation zwischen Generalanwälten und Richtern dahingehend, dass ein Fall immer im Tandem vom Bericht erstattenden Richter und zuständigen Generalanwalt betreut wird. So müssen Bericht erstattender Richter und zuständiger Generalanwalt z.B. der Generalversammlung des Gerichtshofs vorschlagen, in welcher Formation ein Fall entschieden wird, in einer Dreierkammer, in einer Fünferkammer, in der Großen Kammer mit 13 Richtern oder, was aber praktisch nicht vorkommt, im Plenum. Zuständiger Generalanwalt und Richter machen regelmäßig einen gemeinsamen Vorschlag. Aber die Generalanwälte nehmen nicht an der Urteilsberatung teil. Unsere Beteiligung an einer Rechtssache ist mit Verkündung unserer Schlussanträge beendet. Die Richter beraten dann, ob sie uns folgen und wie das Urteil im Einzelnen lautet.

\section{Welche Rechtsgebiete bearbeiten Sie hauptsächlich? Gibt es eine Spezialisierung beim EuGH?}

Es gibt keine Spezialisierung beim EuGH. Die dahinter stehende Idee ist, dass alle Rechtsgebiete, die gesamte Rechtsprechung, von allen Mitgliedern des Gerichtshofs getragen wird. So kann es nicht dazu kommen, dass ein Richter ein ganzes Gebiet ohne den Beitrag der anderen Rechtskulturen prägt. Deshalb haben wir, wie so mancher Supreme Court, das Generalistenprinzip. Das bedeutet, dass jeder sich mit allem beschäftigt. Mittlerweile ist „alles“ ziemlich viel, weil das Europäische Gemeinschaftsrecht quasi sämtliche Bereiche abdeckt. In neuerer Zeit sind viele Aspekte des Zivilrechts und des Strafrechts hinzugekommen. Die Spanne ist sehr weit. Immer mal wieder gibt es die Diskussion, ob man Spezialzuständigkeiten einführen sollte, vielleicht mit zunehmender Berechtigung.

\section{Wie sieht eine normale Arbeitswoche der deutschen Gene-} ralanwältin am Europäischen Gerichtshof aus?

Montag ist regelmäßig der Tag der protokollarischen Anlässe. Oft gibt es Besuche von Delegationen oberster Gerichte aus den Mitgliedstaaten oder auch mal aus einem Ministerium, mit denen man Rechtsfragen diskutiert. Dienstags ist morgens oft mündliche Verhandlung, so wie auch heute, von halb zehn fast bis halb zwei. Meistens füllen die mündlichen Verhandlungen nur einen Vormittag aus, ganz selten gehen sie auch mal bis weit in den Nachmittag hinein. Dienstagabend ist immer Generalversammlung des Gerichtshofs, in der entschieden wird, welche Rechtssache welcher Kammer zugeordnet wird. Mittwochmorgens ist vielleicht auch eine mündliche Verhandlung, genauso wie donnerstags. Aber es ist die Ausnahme, drei mündliche Verhandlungen in einer 
Woche zu haben. Es kann auch nur eine sein. Weiter gibt es noch die Besprechungen und Vorbereitungen mit den Mitarbeitern oder Besprechungen mit anderen Kollegen. Hauptaufgabe bleibt natürlich das Verfassen der Schlussanträge, und man muss darauf achten, dass ununterbrochene Zeit zum Nachdenken, Recherchieren und Formulieren verbleibt. Freitags ist ein Tag, an dem meistens keine Termine für Generalanwälte stattfinden. Die Richter beraten Freitagmorgen, die Generalanwälte nicht. Den Generalanwälten bleibt dann Zeit, sich auf das Verfassen ihrer Schlussanträge zu konzentrieren.

Haben Sie den Wechsel von der Universität zum EuGH nach Luxemburg als große Umstellung empfunden? Welche Aspekte Ihrer Tätigkeit als Universitätsprofessorin bzw. als Generalanwältin schätzen Sie besonders?

Der Wechsel von der Universität an den Gerichtshof war nicht sehr drastisch. Denn gerade die Tätigkeiten einer Generalanwältin und Universitätsprofessorin weisen Gemeinsamkeiten auf. So muss man beim Verfassen von Schlussanträgen neue Rechtsfragen erforschen. Ich muss sogar sagen, dass das Leben oft noch viel interessantere Fragestellungen birgt, als man sie sich als Universitätslehrerin im Rahmen von Aufsätzen ausdenken könnte. Die Forschung wird hier also in gewisser und sehr vielfältiger Weise fortgeführt. Demgegenüber wurde die Lehre ganz kräftig zurückgedrängt: Ich halte hie und da einen Vortrag, lehre aber nur noch völlig marginal, weil ich quasi alle Tage hauptberuflich als Generalanwältin beschäftigt bin. Nur ab und zu gebe ich eine kurze Lehrveranstaltung am Collège d'Europe in Brügge und oder eine Blockveranstaltung an der Universität St. Gallen.

Wir haben schon erfahren, wie prall gefüllt Ihr Terminkalender ist und wie diszipliniert und gut organisiert Sie Ihren Arbeitsalltag bewältigen. Wie entspannen Sie sich und tanken neue Energie?

Ich bin gerne im Freien und treibe dort Sport, etwa im Winter Skifahren. Unsere Kinder sind mittlerweile teils schon wahre Rennfahrer, da muss ich sehen, dass ich überhaupt noch mitkomme, aber es macht sehr viel Spaß. Oder wir spielen Tennis. So lässt sich manche Entspannungstätigkeit mit Familienleben verbinden. Ansonsten lese ich gerne oder mein Mann und ich gehen aus. Anlässlich meines aktuellen Geburtstages machen wir einen „kleinen“ Betriebsausflug in Form einer nachmittäglichen Wanderung in Luxemburg.

Und nun zur letzten Frage: Sie sind schon seit langen Jahren djb-Mitglied. Was schätzen Sie am djb?

Am djb schätze ich das Kennenlernen von und die Kommunikation mit hochqualifizierten und sympathischen Kolleginnen sowie das Netzwerk.

Vielen Dank Frau Professorin Kokott, dass Sie sich die Zeit genommen haben für dieses Interview.

\section{Impressum}

\author{
Schriftleitung: \\ Anke Gimbal, Rechtsassessorin (V.i.S.d.P.) \\ Juliane Lindner
}

Redaktionsanschrift:

Deutscher Juristinnenbund e. V.

Anklamer Str. 38

10115 Berlin

Telefon: 030 443270-0

Telefax: 030 443270-22

E-Mail: geschaeftsstelle@djb.de

\section{Druck und Verlag:}

Nomos Verlagsgesellschaft mbH \& Co. KG

Waldseestr. 3-5

D-76530 Baden-Baden

Telefon: 07221 2104-O

Telefax: 07221 2104-27

\author{
Anzeigenverwaltung und \\ Anzeigenannahme: \\ Sales friendly, Verlagsdienstleistungen \\ Bettina Roos \\ Siegburgerstr. 123 \\ D-53229 Bonn \\ Telefon: 0228 97898-o \\ Telefax: 0228 97898-20 \\ E-Mail: roos@sales-friendly.de
}

Die Zeitschrift sowie alle in ihr enthaltenen einzelnen Beiträge und Abbildungen sind urheberrechtlich geschützt. Jede Verwertung, die nicht ausdrücklich vom Urheberrechtsgesetz zugelassen ist, bedarf der vorherigen Zustimmung des Verlages.

Namentlich gekennzeichnete Artikel müssen nicht die Meinung des Herausgebers oder der Schriftleitung wiedergeben. Unverlangt eingesendete Manuskripte - für die keine Haftung übernommen wird - gelten als Veröffentli- chungsvorschlag zu den Bedingungen des Verlags. Es werden nur unveröffentlichte Originalarbeiten angenommen. Die Verfasser erklären sich mit einer nicht sinnentstellenden redaktionellen Bearbeitung einverstanden.

\author{
Erscheinungsweise: \\ vierteljährlich \\ Bezugspreis 2009: \\ jährlich $48,-€$, Einzelheft $13,-€$
}

Alle Preise zzgl. Vertriebs-Direktbeordnungsgebühren inkl. MWSt.;

Bestellungen nehmen entgegen:

Der Buchhandel und der Verlag; Kündigung jeweils drei Monate zum Kalenderjahresende. Zahlungen jeweils im Voraus an: Nomos Verlagsgesellschaft, Postbank Karlsruhe, Konto 7363651 (BLZ 66010075) oder Stadtsparkasse Baden-Baden, Konto 5002266 (BLZ 66250030). 